\title{
Partial Charges Optimized by Genetic Algorithms for Deep Eutectic Solvent Simulations
}

\author{
Xiang Zhong, Caroline Velez, and Orlando Acevedo* \\ Department of Chemistry, University of Miami, Coral Gables, Florida 33146 \\ E-mail: orlando.acevedo@miami.edu
}

\section{Supporting Material.}

Table S1. GAML parameterization of benzene using CHELPG.............................

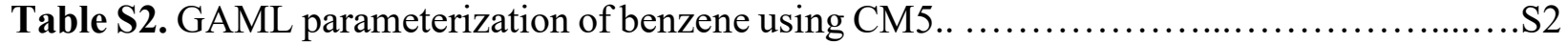

GAML software is available for download at:

https://github.com/orlandoacevedo/GAML

mLJR software is available for download at:

https://github.com/orlandoacevedo/mLJR

GROMACS formatted force field parameter files are available for download at:

https://github.com/orlandoacevedo/DES

Table S1. Six separate GAML parameterization attempts of partial charges for benzene (symmetrical $\mathrm{C}$ and $\mathrm{H}$ atoms) using an identical CHELPG starting point. Percent errors are predictions compared to experimental solvent properties of density $\left(\mathrm{g} / \mathrm{cm}^{3}\right), \Delta H_{\mathrm{vap}}(\mathrm{kcal} / \mathrm{mol})$, and $\Delta G_{\text {hyd }}(\mathrm{kcal} / \mathrm{mol})$.

\begin{tabular}{c|ccc|cc}
\hline $\begin{array}{c}\text { Benzene } \\
\text { Attempt \# }\end{array}$ & Density error & \multicolumn{3}{|c|}{ Percent error } & \multicolumn{2}{c}{ Atomic charges (e) } \\
\hline 1 & $\Delta H_{\text {vap error }}$ & $\Delta G_{\text {hyd }}$ error & C & H \\
2 & $-2.80 \%$ & $-5.04 \%$ & $3.06 \%$ & -0.089 & 0.089 \\
3 & $-2.91 \%$ & $-4.48 \%$ & $5.00 \%$ & -0.093 & 0.093 \\
4 & $-2.93 \%$ & $-5.77 \%$ & $0.28 \%$ & -0.090 & 0.090 \\
5 & $-4.07 \%$ & $-5.83 \%$ & $4.72 \%$ & -0.089 & 0.089 \\
6 & $-3.02 \%$ & $-6.00 \%$ & $6.94 \%$ & -0.091 & 0.091 \\
\hline
\end{tabular}


Table S2. GAML parameterization of partial charges for benzene starting from the CM5 charge model.

\begin{tabular}{cccccc}
\hline Benzene & & \multicolumn{2}{c}{ Percent error } & & \multicolumn{2}{c}{ Atomic charges (e) } \\
\cline { 3 - 6 } CM5 & Density error & $\Delta H_{\text {vap error }}$ & $\Delta G_{\text {hyd error }}$ & $\mathrm{C}$ & $\mathrm{H}$ \\
\hline & $-3.71 \%$ & $-6.53 \%$ & $-3.61 \%$ & -0.091 & 0.091 \\
\hline
\end{tabular}

